\title{
CRITICAL PERCOLATION ON ANY NONAMENABLE GROUP HAS NO INFINITE CLUSTERS
}

\author{
By Itai Benjamini, Russell Lyons, ${ }^{1}$ Yuval Peres ${ }^{2}$ and \\ ODED SCHRAMM ${ }^{3}$ \\ Weizmann Institute of Science, Indiana University, Hebrew University \\ and University of California, Berkeley and Weizmann Institute \\ of Science
}

\begin{abstract}
We show that independent percolation on any Cayley graph of a nonamenable group has no infinite components at the critical parameter. This result was obtained by the present authors earlier as a corollary of a general study of group-invariant percolation. The goal here is to present a simpler self-contained proof that easily extends to quasi-transitive graphs with a unimodular automorphism group. The key tool is a "mass-transport" method, which is a technique of averaging in nonamenable settings.
\end{abstract}

1. Introduction. The main long-standing open question in percolation theory is to show that critical percolation in $\mathbb{Z}^{d}$ has no infinite components for all $d \geq 2$. The work of Harris (1960) and Kesten (1980) established the two-dimensional case; Hara and Slade (1994) proved it for $d \geq 19$. Recently, a study of percolation on other graphs, such as Cayley graphs, was initiated. The relevant definitions appear below. Here is our main theorem.

THEOREM 1.1. Let $X$ be a Cayley graph of a finitely generated nonamenable group, and consider either site or bond percolation on $X$. Then at the corresponding critical value $p=p_{c}$, almost surely there is no infinite component.

In particular, there are no infinite components for critical percolation on any lattice in hyperbolic space, nor on a $(k$-regular tree) $\times \mathbb{Z}$. The latter was previously known when $k \geq 7$ [Wu (1993)]. Wu's proof goes along the lines of the high-dimensional Euclidean proof and uses the triangle condition.

The key tool in our proof is a technique of "mass transport." This tool is especially valuable in nonamenable settings, where it can sometimes replace the ergodic theorem for approximation of expectations by suitable spatial averages.

\footnotetext{
Received November 1997; revised August 1998.

${ }^{1}$ Supported in part by the Institute for Advanced Studies, Jerusalem.

${ }^{2}$ Supported in part by NSF Grant DMS-98-03597.

${ }^{3}$ Supported in part by the Sam and Ayala Zacks Professorial Chair.

AMS 1991 subject classifications. Primary 60B99; secondary 60D05, 82B43.

Key words and phrases. Percolation, Cayley graphs, amenability.
} 
Early forms of the mass-transport method were used by Adams (1990), van den Berg and Meester (1991) and Häggström (1997). The method was developed further in Benjamini, Lyons, Peres and Schramm (1999). The latter paper, denoted BLPS (1999) below, is a study of group-invariant, possibly dependent, percolation models on transitive graphs. After completing the first draft of that paper, we realized that combining two of the results there yields Theorem 1.1.

The resulting proof of Theorem 1.1 was not the most direct possible; in this note, we illustrate the technique of mass transport by presenting the simplest proof we know for Theorem 1.1. Another advantage of the proof given here is that it easily extends to the wider setup of quasi-transitive graphs with a unimodular nonamenable automorphism group. Nevertheless, the present proof still uses invariant percolation models.

In the study of percolation on nonamenable graphs, new phenomena, such as a double phase transition, appear; the first example of this is due to Grimmett and Newman (1990), who considered percolation on the product of a regular tree and $\mathbb{Z}$. Benjamini and Schramm (1996) present an introduction to the subject and a list of problems, some of which have been recently solved; see the final section of the present paper for an update. In that section, we also survey some further results and conjectures concerning percolation on nonamenable graphs.

Terminology. Let $\Gamma$ be a finitely generated group. Given a finite symmetric set of generators $S=\left\{g_{1}^{ \pm 1}, \ldots, g_{n}^{ \pm 1}\right\}$ for $\Gamma$, the (right) Cayley graph of $\Gamma$ is the graph $X(\Gamma, S):=(\mathrm{V}, \mathrm{E})$ with $\mathrm{V}:=\Gamma$ and $[g, h] \in \mathrm{E}$ iff $g^{-1} h \in S$. Thus, the graph $X(\Gamma, S)$ depends on the set of generators. Note that any two Cayley graphs of the same group are roughly isometric (quasi-isometric). More generally, a graph $X$ is called transitive if for any vertices $x, y$, there is a graph automorphism mapping $x$ to $y$. Clearly, any Cayley graph is transitive by the action of left multiplication.

Let the edge-isoperimetric constant of $X$ be

$$
\iota_{E}(X):=\inf \left\{\frac{\left|\partial_{E} K\right|}{|K|} ; K \subset \mathrm{V} \text { is finite }\right\},
$$

where $\partial_{\mathrm{E}} K$, the edge boundary of $K$, consists of all edges $[u, v]$ with $u \in K$ and $v \in \mathrm{V} \backslash K$. A finitely generated group is nonamenable if for some finite generating set, the edge-isoperimetric constant of its Cayley graph is positive. The isoperimetric constant of a graph is sometimes called the Cheeger constant.

In $p$-Bernoulli bond percolation on a graph $X=(\mathrm{V}(X), \mathrm{E}(X))$, the edges are open with probability $p$ independently. Those edges that are not open are closed. The corresponding product measure on the edge configurations is denoted $\mathbf{P}_{p}$. The percolation subgraph is the random graph whose vertices are $\mathrm{V}(X)$ and whose edges are the open edges. Let $K(v)$ be the cluster of $v$, 
that is, the connected component of $v$ in the percolation subgraph. We write

$$
\theta_{v}(p):=\mathbf{P}_{p}[K(v) \text { is infinite }] .
$$

On a Cayley graph (or, more generally, on a transitive graph), the value of $\theta_{v}(p)$ is independent of the choice of $v$, whence the subscript $v$ is dropped. Let

$$
p_{c}:=\inf \{p ; \theta(p)>0\}
$$

be the critical probability for percolation. Bernoulli site percolation is defined similarly with vertices replacing edges. See Grimmett (1989) for more information about Bernoulli percolation.

2. Mass transport. Bernoulli percolation is one example of an invariant percolation model. If a group $\Gamma$ acts by automorphisms on a graph $X$, we call a probability measure on the subgraphs of $X$ a $\Gamma$-invariant percolation model if it is invariant under the action of $\Gamma$. An invariant percolation model $\mathbf{P}$ is a bond percolation model if $\mathbf{P}[\mathrm{V}(\omega)=\mathrm{V}(X)]=1$, where $\omega$ denotes a subgraph of $X$.

We now present the mass-transport principle. Let $m(x, y ; \omega)$ be a nonnegative function of three variables: two vertices $x, y$ and a subgraph $\omega$ of $X$. Intuitively, $m(x, y ; \omega)$ represents the mass transported from $x$ to $y$ when the percolation subgraph is $\omega$. We suppose that $m(\cdot, \cdot ; \cdot)$ is invariant under the diagonal action of $\Gamma$, that is, $m(x, y ; \omega)=m(g x, g y ; g \omega)$ for all $x, y, g$ and $\omega$. The mass-transport principle asserts that for any invariant percolation, the expected total mass transported out of any vertex $x$ equals the expected total mass transported into $x$, that is,

$$
\forall x \in \mathrm{V} \sum_{y \in \mathrm{V}} M(x, y)=\sum_{y \in \mathrm{V}} M(y, x),
$$

where $M(x, y):=\mathbf{E} m(x, y ; \omega)$ is also invariant under the diagonal action of $\Gamma$.

On a Cayley graph, it is straightforward to verify (2.1):

$$
\sum_{y \in \mathrm{V}} M(x, y)=\sum_{g \in \Gamma} M(x, g x)=\sum_{g \in \Gamma} M\left(g^{-1} x, x\right)=\sum_{y \in \mathrm{V}} M(y, x) .
$$

The creative element in applying the mass-transport method is to make a judicious choice of the transport function $m(x, y ; \omega)$. This depends on the particular application; three such applications are given in this note.

The identity (2.1) is valid on many transitive graphs that are not Cayley graphs, provided their automorphism group is unimodular; see BLPS (1999). The proof of Theorem 1.1 below uses only transitivity of $X$ and (2.1), so it applies to these graphs as well. With minor modifications, the proof also applies to unimodular quasi-transitive graphs, that is, graphs $X=(\mathrm{V}, \mathrm{E})$ for which there is some unimodular group $\Gamma$ of automorphisms with finitely many orbits in V.

Let $S$ be a finite set of generators for a nonamenable group $\Gamma$ and let $X:=X(\Gamma, S)$ be the corresponding Cayley graph. For a finite subgraph $K \subset X$, 
set

$$
\alpha_{K}:=\frac{1}{|\mathrm{~V}(K)|} \sum_{x \in \mathrm{V}(K)} \operatorname{deg}_{K}(x),
$$

where $\operatorname{deg}_{K}(x)$ refers to the degree of $x$ as a vertex in the graph $K$. Let

$$
\alpha(X):=\sup \left\{\alpha_{K} ; K \subset X \text { is finite }\right\} .
$$

Since every vertex of $X$ has the same degree, it follows that

$$
\alpha(X)+\iota_{\mathrm{E}}(X)=\operatorname{deg}_{X}(o),
$$

where $o \in \mathrm{V}(X)$ is the identity of $\Gamma$.

THEOREM 2.1. Let $X$ be a Cayley graph of a nonamenable group $\Gamma$ with respect to some finite set of generators. Let $o \in \mathrm{V}(X)$ denote the identity of $\Gamma$. Suppose that $\xi$ is a random subgraph of $X$ and the distribution of $\xi$ is an invariant bond percolation model on $X$. If $\mathbf{E} \operatorname{deg}_{\xi}(o)>\alpha(X)$, then $\xi$ has an infinite component with positive probability. tails.

REMARK 2.2. It suffices that $\mathbf{E} \operatorname{deg}_{\xi}(o) \geq \alpha(X)$. See BLPS (1999) for de-

Corollary 2.3. If $\mathbf{P}[e \notin \xi]<\iota_{\mathrm{E}}(X) / \operatorname{deg}_{X}(o)$ for all $e \in \mathrm{E}(X)$, then $\xi$ has an infinite component with positive probability.

The proof follows immediately from Theorem 2.1 and (2.2).

Proof of Theorem 2.1. For each $v \in \mathrm{V}(X)$, let $K(v)$ denote the component of $\xi$ containing $v$. Set

$$
m(v, u ; \xi):= \begin{cases}\operatorname{deg}_{\xi}(v) /|K(v)|, & \text { if } K(v) \text { is finite and } u \in K(v), \\ 0, & \text { otherwise. }\end{cases}
$$

Thus,

$$
\sum_{u \in \mathrm{V}(X)} m(u, o ; \xi)=\alpha_{K(o)} \leq \alpha(X)
$$

whenever $K(o)$ is finite, and $\Sigma_{u \in \mathrm{V}(X)} m(u, o ; \xi)=0$ otherwise. On the other hand,

$$
\sum_{u \in \mathrm{V}(X)} m(o, u ; \xi)= \begin{cases}\operatorname{deg}_{\xi}(o), & \text { when } K(o) \text { is finite, } \\ 0, & \text { otherwise. }\end{cases}
$$

Since $m(\cdot, \cdot ; \cdot)$ is diagonally invariant, the mass-transport principle gives

$$
\begin{aligned}
& \mathbf{E}\left[\operatorname{deg}_{\xi}(o)|| K(o) \mid<\infty\right] \mathbf{P}[|K(o)|<\infty] \\
& \quad=\mathbf{E} \sum_{u \in \mathrm{V}(X)} m(o, u ; \xi)=\mathbf{E} \sum_{u \in \mathrm{V}(X)} m(u, o ; \xi) \leq \alpha(X) \mathbf{P}[|K(o)|<\infty] .
\end{aligned}
$$

When $\mathbf{P}[|K(o)|<\infty]=1$, we get $\mathbf{E} \operatorname{deg}_{\xi}(o) \leq \alpha(X)$, as required. 


\section{Ruling out a unique infinite cluster.}

Proof OF Theorem 1.1. For simplicity, we restrict our attention to critical bond percolation; the proof for critical site percolation requires only minor modifications.

The number of infinite clusters is invariant under the action of $\Gamma$. By ergodicity, the number of infinite clusters is constant a.s. It follows that this constant must be 0,1 or $\infty$; see Newman and Schulman (1981). In this section, we rule out a unique infinite cluster at $p=p_{c}$, and in the next section, we rule out infinitely many infinite clusters.

Denote the graphical distance in $X$ by $d_{X}$, and let $\omega$ be critical Bernoulli bond percolation on $X$. Suppose that almost surely there is a unique infinite cluster $U$ of $\omega$. Let $\gamma_{\varepsilon}$ be an $\varepsilon$-Bernoulli bond percolation on $X$ that is independent of $\omega$. We use $\gamma_{\varepsilon}$ to define a bond percolation model $\xi_{\varepsilon}$ on $X$ as follows. For $v \in \mathrm{V}$, let $U(v) \subset U$ be the set of vertices $u \in U$ such that $d_{X}(v, u)=d_{X}(v, U)$; that is, $U(v)$ is the set of vertices of $U$ that are closest to $v$. Given two neighbors $v, w \in \mathrm{V}$, put the edge $[v, w]$ in $\xi_{\varepsilon}$ iff $d_{X}(v, U)<1 / \varepsilon$, $d_{X}(w, U)<1 / \varepsilon$, and $U(v) \cup U(w)$ is contained in a connected component of $\omega \backslash \gamma_{\varepsilon}$. If we fix $\omega$, then

$$
\lim _{\varepsilon \rightarrow 0} \mathbf{P}\left[[v, w] \notin \xi_{\varepsilon} \mid \omega\right]=0,
$$

because every pair of vertices in $U$ is joined by some finite path in $U$ that is unlikely to intersect $\gamma_{\varepsilon}$ when $\varepsilon$ is small. Consequently, the bounded convergence theorem gives

$$
\lim _{\varepsilon \rightarrow 0} \mathbf{P}\left[[v, w] \notin \xi_{\varepsilon}\right]=0 .
$$

By Corollary 2.3, it follows that for $\varepsilon>0$ sufficiently small, with positive probability, there will be an infinite component of $\xi_{\varepsilon}$. However, when there is an infinite $\xi_{\varepsilon}$-component, there must be an infinite component in $\omega \backslash \gamma_{\varepsilon}$. As $\omega \backslash \gamma_{\varepsilon}$ is a $(1-\varepsilon) p_{c}$-Bernoulli bond percolation, this contradicts the definition of $p_{c}$ and proves that $\omega$ cannot have a unique infinite cluster.

4. Ruling out multiple infinite clusters. In this section, we continue with the proof of Theorem 1.1, dealing with the case that critical percolation produces more than one infinite cluster.

Definition 4.1. Let $G$ be an infinite graph. A vertex $v \in \mathrm{V}(G)$ is an encounter point if there are three or more neighbors of $v$ that belong to distinct infinite connected components of $G \backslash\{v\}$.

Suppose that with positive probability, $\omega$ has more than one infinite cluster. It is then well known [Burton and Keane (1989)] that the set $Y$ of encounter points of $\omega$ is nonempty almost surely. 
We say that $C$ has an w-neighbor of $v$ if there is some $x \in C$ with $[x, v] \in \omega$.

Lemma 4.2. Consider Bernoulli bond percolation $\omega$ with $p \geq p_{c}$ on $a$ Cayley graph. With $\mathbf{P}_{p}$-probability 1 , for every encounter point $v$ of $\omega$ and every infinite component $C$ of $\omega \backslash\{v\}$ having an $\omega$-neighbor of $v$, there is some encounter point of $\omega$ in $C$.

Proof. Let $u, v \in \mathrm{V}$. Set $m(u, v ; \omega):=1$ if $u$ and $v$ are in the same cluster of $\omega$ and $v$ is the unique encounter point of $\omega$ with minimal distance to $u$ (where distances are measured in $\omega$ ). Otherwise, set $m(u, v ; \omega):=0$. Then $\Sigma_{u \in \mathrm{V}} m(v, u ; \omega) \leq 1$. By the mass-transport principle, it follows that almost surely $\sum_{u \in \mathrm{V}} m(u, v ; \omega)<\infty$ for all $v \in \mathrm{V}$. This implies the lemma.

We now construct an invariant forest $\xi$ whose vertices are $Y$, the encounter points of $\omega$. The edges of $\xi$ will not necessarily be in E. For each $v \in \mathrm{V}$, let $x(v)$ be a uniformly distributed random variable in $[0,1]$ such that $\omega$ and all $x(v)$ are mutually independent. Suppose that $v \in Y$. For every infinite connected component $Z$ of $\omega \backslash\{v\}$ having an $\omega$-neighbor of $v$, let $Y(Z, v) \subset Y \cap Z$ be the set of encounter points of $\omega$ in $Z$ that are closest (in $\omega)$ to $v$, and let $u(Z, v)$ be the vertex $u \in Y(Z, v)$ that minimizes $x(u)$. Let $\xi$ be the set of all edges $[v, u(Z, v)]$, where $v \in Y$ and $Z$ is an infinite connected component of $\omega \backslash\{v\}$ having an $\omega$-neighbor of $v$. It is clear that $\xi$ is an invariant graph on $\mathrm{V}$. For every $v \in Y$, the degree of $v$ in $\xi$ is at least 3 by Lemma 4.2.

We now verify that $\xi$ is almost surely a forest; that is, it contains no cycles. Assume otherwise, and suppose that $C$ is a cycle in $\xi$ of length greater than or equal to 3 . When $u=u(Z, v)$, consider edges in $\xi$ of the form $[v, u]$ to be directed from $v$ to $u$. Let $v$ be a vertex in $C$ and note that $C \backslash\{v\}$ is contained in some connected component $Z$ of $\omega \backslash\{v\}$, because $v$ appears only once in $C$. This shows that the number of edges in $C$ that are directed away from $v$ is at most 1 . Since the number of edges in $C$ equals the number of vertices in $C$, every vertex must have one edge directed toward it and one edge directed away from it. If $[a, b]$ and $[b, c]$ are directed edges in $C$, then the distance in $\omega$ from $b$ to $c$ cannot be larger than the distance from $a$ to $b$. As $C$ is a cycle, it follows that these distances must be the same. Let $z$ be the vertex in $C$ that maximizes $x(z)$. Then there must be vertices $a, b$ in $C$ such that $[a, b]$ and $[b, z]$ are directed edges of $C$ and $a \neq z$. But $a$ and $z$ are at the same distance from $b$ and $x(a)<x(z)$. This contradicts the existence of the directed edge $[b, z]$ in $C$, and this contradiction proves that $\xi$ is a forest.

Let $\varepsilon>0$ and let $\gamma_{\varepsilon}$ be an $\varepsilon$-Bernoulli bond percolation process. Since $\omega$ is critical, almost surely $\omega \backslash \gamma_{\varepsilon}$ has only finite components. Now define an invariant bond percolation model $\xi_{\varepsilon} \subset \xi$ as follows. For any edge $[v, u] \in \xi$, let $[v, u] \in \xi_{\varepsilon}$ iff $v$ and $u$ are in the same component of $\omega \backslash \gamma_{\varepsilon}$. Then almost surely $\xi_{\varepsilon}$ has only finite components.

We now perform mass transport on $\mathrm{V}$. For any $v \in \mathrm{V}$, let $K(v)$ denote its $\xi_{\varepsilon}$-connected component (which is almost surely finite) and let $K_{\partial}(v)$ denote 
the set of vertices $u \in K(v)$ that have some $\xi$-neighbor outside of $K(v)$. Set

$$
m(v, u ; \xi):= \begin{cases}1 /\left|K_{\partial}(v)\right|, & \text { if } v \in Y \text { and } u \in K_{\partial}(v), \\ 0, & \text { otherwise. }\end{cases}
$$

By the mass-transport principle, we know that

$$
\sum_{u \in \mathrm{V}} \mathbf{E}[m(v, u ; \xi)]=\sum_{u \in \mathrm{V}} \mathbf{E}[m(u, v ; \xi)]
$$

Note that

$$
\sum_{u \in \mathrm{V}} m(u, v ; \xi)= \begin{cases}|K(v)| /\left|K_{\partial}(v)\right|, & \text { when } v \in Y \cap K_{\partial}(v), \\ 0, & \text { otherwise. }\end{cases}
$$

Because $\xi$ is a forest and each vertex in $Y$ has $\xi$-degree at least 3, it easily follows that $2\left|K_{\partial}(v)\right| \geq|K(v)|$. Consequently, (4.2) shows that $\sum_{u \in \mathrm{V}} m(u, v ; \xi)$ $\leq 2$ almost surely. On the other hand, $\Sigma_{u \in v} m(v, u ; \xi)$ is 1 if $v \in Y$ and 0 otherwise. Therefore, (4.1) and (4.2) give

$$
\mathbf{P}[v \in Y] \leq 2 \mathbf{P}\left[v \in Y, v \in K_{\partial}(v)\right] .
$$

If we fix $\omega, \xi$, and $e \in \xi$, then the probability that $e \notin \xi_{\varepsilon}$ tends to 0 as $\varepsilon \rightarrow 0$ : there is some finite path in $\omega$ joining the endpoints of $e$, and when $\varepsilon$ is small, this path is unlikely to meet $\gamma_{\varepsilon}$. From the bounded convergence theorem, it therefore follows that for any $v \in \mathrm{V}$, the probability that $v$ has some edge in $\xi \backslash \xi_{\varepsilon}$ tends to 0 as $\varepsilon \rightarrow 0$. In other words,

$$
\lim _{\varepsilon \rightarrow 0} \mathbf{P}\left[v \in Y, v \in K_{\partial}(v)\right]=0 .
$$

This contradicts (4.3) since $\mathbf{P}[v \in Y]$ is positive and does not depend on $\varepsilon$. Hence the proof of Theorem 1.1 is complete.

\section{Further results and problems on percolation in nonamenable} groups. Recall that for percolation on $\mathbb{Z}^{d}$, if $\theta(p)>0$, then there exists with probability 1 a unique infinite open cluster. [See Aizenman, Kesten and Newman (1987) and the elegant proof in Burton and Keane (1989).] As was shown by Grimmett and Newman (1990), for percolation on a ( $k$-regular tree) $\times \mathbb{Z}$, this is not the case if $k$ is sufficiently large: for some values of $p$, uniqueness holds, while for others, there are infinitely many infinite clusters. Benjamini and Schramm (1996) defined

$$
p_{u}:=\inf \left\{p ; \mathbf{P}_{p} \text { [there is exactly one infinite cluster] }=1\right\}
$$

and conjectured that $p_{c}<p_{u}$ for percolation on nonamenable Cayley graphs. This was established by Lalley (1996) for certain planar Cayley graphs, then by Benjamini and Schramm (1998a) for all nonamenable planar Cayley graphs. Häggström and Peres (1998), in response to another question from Benjamini and Schramm (1996), showed that for any $p>p_{u}$, uniqueness holds almost surely. Benjamini and Schramm (1996) also asked whether there is uniqueness at $p_{u}$ in transitive nonamenable graphs. This is true when the graphs are planar [Benjamini and Schramm (1998a)], but Schonmann (1998) has shown it to be false for ( $k$-regular tree) $\times \mathbb{Z}$ when $k \geq 3$. We 
note that for Cayley graphs of finitely presented groups with one end, Babson and Benjamini (1999) showed that $p_{u}<1$. A brief description of some recent developments concerning percolation on general graphs is presented by Benjamini and Schramm (1998b).

RemarK 5.1. Lemma 4.2 has the following consequence. Consider Bernoulli percolation $\omega$ with $p>p_{c}$ on a Cayley graph, and suppose that $\omega$ has more than one infinite cluster a.s. Then with probability 1 , any infinite cluster $C$ of $\omega$ has infinitely many encounter points. Consequently, the space of ends of $C$ is topologically a Cantor set (in particular, it is uncountable). The same proof works for unimodular quasi-transitive graphs. The result for all quasi-transitive graphs was conjectured by Benjamini and Schramm (1996) and first established by Häggström and Peres (1998) in the unimodular case, using a more complicated argument. Very recently, this result on ends was extended to the nonunimodular case by Häggström, Peres and Schonmann (1998). Their argument is based on different principles; in particular, it is not known in the nonunimodular case whether for $p \in\left(p_{c}, p_{u}\right)$, every infinite cluster must contain infinitely many encounter points.

Theorem 1.1 extends easily to some other percolation models; for example, if different (positive) retention probabilities are assigned to different generators in a nonamenable Cayley graph $X$, then for parameter values on the corresponding critical submanifold, there are no infinite clusters in $X$.

To analyze the Ising model on nonamenable groups, it would be useful to know whether Theorem 1.1 also extends to the FK random cluster model for $q \leq 2$; see, for example, Grimmett (1995) for definitions and background. For $q>2$, the (wired) random cluster model on a regular tree has infinite clusters at criticality; see Häggström (1996).

Combining Theorem 1.1 with a result of Häggström and Peres (1998) gives that for nonamenable Cayley graphs, the function $\theta(p)=\mathbf{P}_{p}[K(o)$ is infinite $]$ is continuous for all $p \in[0,1]$. One may wish for quantitative versions of this result, for example as in the following.

Problem 5.1. Bound the modulus of continuity of $\theta(\cdot)$ for nonamenable Cayley graphs. Is $\sup _{p>p_{c}} \theta^{\prime}(p)<\infty$ ? $=0$.

Since $\theta\left(p_{c}\right)=0$, the component $K(o)$ satisfies $\lim _{R \rightarrow \infty} \mathbf{P}_{p_{c}}[\operatorname{diam} K(o)>R]$

Problem 5.2. For a nonamenable Cayley graph $X$, find a bound for $\mathbf{P}_{p_{c}}[\operatorname{diam} K(o)>R]$ that tends to zero as $R \rightarrow \infty$.

It seems reasonable that such a bound would involve only the isoperimetric constant of $X$ and perhaps the degree of the vertices.

Let $\tau_{p}(o, v)$ denote the connectivity function, that is, the $\mathbf{P}_{p}$-probability that $o$ and $v$ are in the same cluster. 
Problem 5.3. For a nonamenable Cayley graph $X$, find a bound for $\tau_{p_{c}}(o, v)$ that goes to zero as $d_{X}(o, v) \rightarrow \infty$.

We expect that $\tau_{p_{c}}(o, v) \leq \alpha^{d_{X}(o, v)}$ for some $\alpha<1$. Lyons and Schramm (1998) have recently shown that on Cayley graphs, $\inf _{v} \tau_{p}(o, v)=0$ when there is no uniqueness at $p$. Schramm (unpublished) has also shown that for every nonamenable Cayley graph $G$, there is an $\alpha<1$ with $\tau_{p_{c}}(o, v)<\alpha^{d_{X}(o, v)}$ for infinitely many $v$.

\section{REFERENCES}

Adams, S. (1990). Trees and amenable equivalence relations. Ergodic Theory Dynamic Systems $101-14$.

Aizenman, M., Kesten, H. and Newman, C. M. (1987). Uniqueness of the infinite cluster and continuity of connectivity functions for short and long range percolation. Comm. Math. Phys. 111 505-531.

Babson, E. and Benjamini, I. (1999). Cut sets and normed cohomology with applications to percolation. Proc. Amer. Math. Soc. 127 589-597.

Benjamini, I., Lyons, R., Peres, Y. and Schramm, O. (1999). Group-invariant percolation on graphs. Geom. Funct. Anal. 9 29-66.

Benjamini, I. and Schramm, O. (1996). Percolation beyond $\mathbb{Z}^{d}$, many questions and a few answers. Electronic Comm. Probab. 1 71-82.

Benjamini, I. and Schramm, O. (1998a). Percolation in the hyperbolic plane. Unpublished manuscript.

Benjamini, I. and Schramm, O. (1998b). Recent progress on percolation beyond $\mathbb{Z}^{d}$. http://www.wisdom.weizmann.ac.il/ ${ }^{\text {schramm/papers/pyond-rep/. }}$

Burton, R. M. and Keane, M. (1989). Density and uniqueness in percolation. Comm. Math. Phys. 121 501-505.

Grimmett, G. R. (1989). Percolation. Springer, New York.

GRIMmeTt, G. R. (1995). The stochastic random-cluster process and the uniqueness of randomcluster measures. Ann. Probab. 23 1461-1510.

Grimmett, G. R. and Newman, C. M. (1990). Percolation in $\infty+1$ dimensions. In Disorder in Physical Systems (G. R. Grimmett and D. J. A. Welsh, eds.) 219-240. Clarendon Press, Oxford.

HÄGGSTRÖM, O. (1996). The random-cluster model on a homogeneous tree. Probab. Theory Related Fields 104 231-253.

HäGGSTRÖM, O. (1997). Infinite clusters in dependent automorphism invariant percolation on trees. Ann. Probab. 25 1423-1436.

HäGGSTRÖM, O. and PERES, Y. (1998). Monotonicity of uniqueness for percolation on transitive graphs: all infinite clusters are born simultaneously. Probab. Theory Related Fields 113 273-285.

Häggström, O., Peres, Y. and Schonmann, R. (1998). Percolation on transitive graphs as a coalescent process: relentless merging followed by simultaneous uniqueness. In Perplexing Probability Problems: Papers in Honor of H. Kesten (M. Bramson and R. Durrett, eds.). Birkhäuser, Boston. To appear.

Hara, T. and Slade, G. (1994). Mean-field behaviour and the lace expansion. In Probability and Phase Transition (G. R. Grimmett, ed.) 87-122. Kluwer, Dordrecht.

HARRIS, T. E. (1960). A lower bound for the critical probability in a certain percolation process. Proc. Cambridge Philos. Soc. 56 13-20.

Kesten, H. (1980). The critical probability of bond percolation on the square lattice equals $\frac{1}{2}$. Comm. Math. Phys. 74 41-59.

Lalley, S. P. (1996). Percolation on Fuchsian groups. Ann. Inst. H. Poincaré Probab. Statist. 34 151-178. 
Lyons, R. and Schramm, O. (1998). Indistinguishability of percolation clusters. Ann. Probab. To appear.

Newman, C. M. and Schulman, L. S. (1981). Infinite clusters in percolation models. J. Statist. Phys. 26 613-628.

Schonmann, R. H. (1998). Percolation in $\infty+1$ dimensions at the uniqueness threshold. In Perplexing Probability Problems: Papers in Honor of H. Kesten (M. Bramson and R. Durrett, eds.). Birkhäuser, Boston. To appear.

VAN DEN BERG, J. and MEESTER, R. W. J. (1991). Stability properties of a flow process in graphs. Random Structures Algorithms 2 335-341.

Wu, C. C. (1993). Critical behavior of percolation and Markov fields on branching planes. $J$. Appl. Probab. 30 538-547.

I. BENJAMINI

Mathematics Department

Weizmann Institute of Science

REHOVOT 76100

ISRAEL

E-MAIL: itai@wisdom.weizmann.ac.il

Y. PERES

INSTitute OF MATHEMATICS

HEBREW UNIVERSITY

GIVAT RAM, JERUSALEM 91904

ISRAEL

AND

Department of Statistics

UNIVERSITY OF CALIFORNIA

Berkeley, California 94720-3860

E-MAIL: peres@stat.berkeley.edu
R. LYONS

Department of MATHEMATics

INDIANA UNIVERSITY

BLOOMINGTON, INDIANA 47405-5701

E-MAIL: rdlyons@indiana.edu
O. SCHRAMM

Mathematics Department

Weizmann Institute of Science

REHOVOT 76100

ISRAEL

E-MAIL:schramm@wisdom.weizmann.ac.il 